\section{Event-based Recursive Distributed Filtering over Wireless Sensor Networks}

\author{
Qinyuan Liu, Zidong Wang, Xiao He and D. H. Zhou
}

\begin{abstract}
In this paper, the distributed filtering problem is investigated for a class of discrete time-varying systems with an event-based communication mechanism. Each intelligent sensor node transmits the data to its neighbors only when the local innovation violates a predetermined Sendon-Delta (SoD) data transmission condition. The aim of the proposed problem is to construct a distributed filter for each sensor node subject to sporadic communications over wireless networks. In terms of an event indicator variable, the triggering information is utilized so as to reduce the conservatism in the filter analysis. An upper bound for the filtering error covariance is obtained in form of Riccati-like difference equations by utilizing the inductive method. Subsequently, such an upper bound is minimized by appropriately designing the filter parameters iteratively, where a novel matrix simplification technique is developed to handle the challenges resulting from the sparseness of the sensor network topology and filter structure preserving issues. The effectiveness of the proposed strategy is illustrated by a numerical simulation.
\end{abstract}

Index Terms-Distributed filtering, wireless sensor networks, eventbased mechanism, Send-on-Delta concept.

\section{INTRODUCTION}

A wireless sensor network is composed of a large number of sensor nodes geographically distributed in certain areas. Generally, the sensors involved in the networks are intelligent nodes with limited computation capability and constrained power supply, and they communicate with each other via wireless communication channels. In the past decades, wireless sensor networks have attracted an increasing attention from researchers due primarily to their attractive application insights in a variety of real-world situations including environmental monitoring, interactive virtual worlds, warehouse inventory and integrated patient monitoring, see [18] for a survey.

An important practical problem with the wireless sensor networks is how to find distributed estimators or filters to extract the information about the state vectors of the target plants from observations contaminated with external disturbances. It is generally known that the traditional Kalman filter algorithm [1], [2], [6], [21], [22] is a recursive least mean square (LMS) one dealing with a single node and is optimal for linear systems with exact system models. On the other hand, to make use of the spatial information of the sensor nodes, distributed filtering problems have recently gained much research attention. Different from the traditional single node, in the distributed filtering schemes, the local filters estimate the system state based on the information not only from itself but also from its neighboring sensors according to the topology of networks. Up to now, many important results have been reported in the literature concerning the distributed filters and several effective strategies have been proposed, see e.g. [3], [5], [8], [11], [12], [17], [19]. Among others, the $H_{\infty}$ filter performance has gained particular research attention [3], [5], [11], [19] due to its capability of ensuring certain worst-case performance with respect to external disturbances. In [12], the robust distributed state estimation problem has been addressed for

This work is supported by National Basic Research Program of China (973 Program) under Grant 2010CB731800, National Natural Science Foundation of China under Grants 61210012, 61290324, 61473163 and 61273156, and Jiangsu Provincial Key Laboratory of E-business at Nanjing University of inance and Economics of China under Grant JSEB201301.

Q. Liu, X. He and D. H. Zhou are with the Department of Automation, Tsinghua University, Beijing 100084, P. R. China.

$\mathrm{Z}$. Wang is with the Department of Computer Science, Brunel University London, Uxbridge, Middlesex, UB8 3PH, United Kingdom. Email: Zidong. Wangebrunel.ac.uk. stochastic sensor network systems based on the mean square error analysis method. Furthermore, in [17], the distributed Kalman filter algorithm has been developed using dynamic consensus protocols with applications to the target tracking problems.

It is worth pointing out that, most available results on distributed filtering problems have implicitly adopted the periodic communication strategy whose communication interval is designed a priori to guarantee desirable performance under the worst conditions. For some engineering systems where the communication bandwidth is not a concern, the periodic strategy could be an acceptable one for its simplicity in system analysis and design. Such a communication strategy, however, would probably lead to many unnecessary transmissions and therefore cause a waste of bandwidth resources. For example, in the case of wireless sensor networks communication constraints, frequent exchanges of signals would inevitably give rise to serious network congestions, which might further induce networkrelated adverse phenomena such as packet losses and communication delays.

In recent years, there have been a growing number of results reported on event-based schedules whose aim is to decrease the unnecessary executions of the systems [14]. In an earlier work [26], the event-based sampling strategy has been compared with the timedriven one and it has been concluded that the former gave better performance for some simple systems. Later, much research efforts has been devoted to the applications of the event-based strategy to various engineering systems such as networked control systems [7], [23], [24] and multi-agent systems [4], [9], [25]. Unfortunately, when it comes to the state estimation problems, the available results in the literature have been scattered, most of which have focused on the practical implementation issues and there is a lack of adequate investigation on systematic analysis on the filtering performance. To be specific, a Send-on-Delta $(\mathrm{SoD})$ regulation has been proposed in [15] for triggering mechanism whose effectiveness against timedriven sampling has been fully discussed. In [13], a modified Kalman filter has been investigated with intermittent measurement updates under the framework of event-based sampling. Another estimation problem with a SoD sampling strategy has been addressed in [16] without considering the communication delays and packet losses. In [20], the hybrid update strategy has been considered to reduce the estimation error based on the assumption that the difference between the present and the latest measurement values is limited within a bounded subset of the measurement space when no event is triggered. Besides, the event-based $H_{\infty}$ filtering problems have been addressed in [10] for continuous-time systems with transmission delays.

Summarizing the above discussion, although the event-based estimation/filtering problem has stirred some initial research attention, the corresponding distributed filtering problem over wireless sensor networks has not yet been adequately investigated due probably to the difficulties in accommodating the topological information of the sensor networks. Note that the sparseness of the sensor network topology and the structure of the distributed filters add substantial challenges to the filter analysis and design, not to mention the difficulties brought from the event-based mechanism, especially when the filtering error variances are required to be locally minimized. As such, we are motivated to deal with the distributed filtering problem over wireless sensor networks according to the event-based strategy by employing a SoD concept. The employed SoD principle ensures that the executions are triggered if certain signal exceeds a timeindependent threshold defined as an important change of its value, and therefore the communication load and energy consumption in practice can be alleviated by avoiding unnecessary transmissions. With the proposed strategy, the sensor broadcasts its information to the neighboring nodes only when the function of its local innovation 
exceeds a fixed time-independent threshold. Obviously, our proposed strategy would reduce the number of transmissions over the wireless communication channel, thereby saving the resource.

The main contributions can be highlighted as follows: 1) a distributed filter is proposed for discrete time-varying systems in the framework of a novel event-based communication protocol; 2) a new event indicator variable is introduced to reflect the triggering information in the filter analysis with hope to reduce possible conservatism in the filter analysis; 3) an upper bound of the filtering error variance is obtained by a recursive algorithm; and 4) the obtained upper bound is minimized at each iteration with proper filter parameters computed via a simplified matrix approach.

Notations. Throughout the paper, the notation used is fairly standard. $\mathbb{R}^{n}$ and $\mathbb{R}^{n \times m}$ denote the $n$-dimensional Euclidean space and the set of all $n \times m$ matrices, respectively. The superscript " $\mathrm{T}$ " denotes the transpose and the notation $X \geq Y$ (respectively, $X>Y$ ), where $\mathrm{X}$ and $\mathrm{Y}$ are symmetric matrices, means that $X-Y$ is positive semidefinite (respectively, positive definite). Furthermore, $\operatorname{diag}_{n}\left\{A_{i}\right\}$ represents the block-diagonal matrix $\operatorname{diag}\left\{A_{1}, \cdots, A_{n}\right\}$. The notation $\operatorname{col}_{n}\left\{x_{i}\right\}$ denotes the column vector $\left\{x_{1}^{T}, \cdots, x_{n}^{T}\right\}^{T}$. $I_{n}$ represents the identity matrix with $n$ rows and $n$ columns. $\mathbb{E}\{x\}$ stands for the expectation of the stochastic variable $x$.

\section{Problem Formulation AND Preliminaries}

Consider a discrete linear time-varying system described by the following state-space model:

$$
x(k+1)=A_{k} x(k)+B_{k} w(k)
$$

where $x(k) \in \mathbb{R}^{n_{x}}$ is the system state and $w(k) \in \mathbb{R}^{n_{w}}$ is the sequence of process noises. $A_{k}, B_{k}$ are known system matrices of appropriate dimensions.

In this paper, a sensor network consisting of $n$ sensor nodes is investigated to measure the output of the target plant. We denote the topology of the network by a fixed directed graph $\mathcal{G}=(\mathcal{V}, \mathcal{E}, \mathcal{H})$ of order $n$ with the set of nodes $\mathcal{V}=\{1,2, \cdots, n\}$, the set of edges $\mathcal{E} \subseteq V \times V$, and the weighted adjacency matrix $\mathcal{H}=\left[a_{i j}\right]$. The weighted adjacency matrix of the graph is a matrix with nonnegative elements $a_{i j}$ satisfying the property $a_{i j}>0 \Longleftrightarrow(i, j) \in \mathcal{E}$, which means that the $i$ th node can receive the information from the $j$ th node. In this case, we can say that the $i$ th nodes is the neighbor of the $j$ th node. Moreover, all the neighbors of node $i$ plus the node itself are denoted by the set as $N_{i}:=\{j \in \mathcal{V} \mid(i, j) \in \mathcal{E}\}$.

For the $i$ th sensor node, the model is described by:

$$
y_{i}(k)=C_{i, k} x(k)+D_{i, k} v(k)
$$

where $y_{i}(k) \in \mathbb{R}^{n_{y}}$ stands for the measurement output received by sensor $i$, and $v(t) \in \mathbb{R}^{n_{v}}$ is the sequence of measurement noises. $C_{i, k}, D_{i, k}$ are known matrices of appropriate dimensions.

Throughout the paper, the following assumptions are made.

Assumption 1: $w(k)$ and $v(k)$ are mutually uncorrelated zeromean Gaussian white-noise sequences with respective covariances $Q_{k}>0$ and $R_{k}>0$.

Assumption 2: The initial state $x(0)$ has the mean $\bar{x}_{0}$ and covariance $P_{0}$, and is uncorrelated with both $w(k)$ and $v(k)$.

\section{A. Traditional distributed filter structure}

Up to now, a large number of results have been developed with respect to the distributed filtering problems over the sensor networks. The traditional distributed filter structures have the general form as follows:

$$
\hat{x}_{i}(k+1)=A_{k} \hat{x}_{i}(k)+\sum_{j \in N_{i}} a_{i j} G_{i j, k} r_{j}(k)
$$

for $i=1,2, \cdots, n$, where $\hat{x}_{i}(k) \in \mathbb{R}^{n_{x}}$ is the estimation of the plant state in the $i$ th sensor node. $G_{i j, k}$ is the filter parameter to be designed. Besides, $r_{i}(k)=y_{i}(k)-C_{i, k} \hat{x}_{i}(k)$ is the innovation sequence exchanged via the network.

So far, to the best of the authors' knowledge, almost all established results for distributed estimation problems basically assume that the sensor nodes should broadcast their local information at every periodic sampling instant. Such a scheme, however, will probably lead to a great deal of unnecessary data flow passing through the network, thereby inevitably aggravating the burden of communication network and increasing the power consumption. In order to significantly improve the network utilization efficiency, in the following, we propose to abandon the traditional periodic paradigm. Instead, we consider a novel event-based mechanism to suitably represent how sensors communicate with each other in a highly efficient way. The main advantage of the modified event-based mechanism lies in its capability of broadcasting the important messages only rather than all messages.

\section{B. Event-based distributed filter structure}

For the purpose of characterizing the SoD triggering mechanism, it is necessary to define event generator functions $f_{i}(.,):. \mathbb{R}^{n_{y}} \times \mathbb{R} \rightarrow$ $\mathbb{R}(i=1, \cdots, n)$ as follows:

$$
f_{i}\left(e_{i}(k), \delta_{i}\right)=e_{i}^{T}(k) e_{i}(k)-\delta_{i} .
$$

Here, $e_{i}(k)=r_{i}^{t}(k)-r_{i}(k)$ where $r_{i}^{t}(k)$ is the broadcast innovation at latest event time, $r_{i}(k)$ is the innovation sequence as defined before, and $\delta_{i}$ is a positive scalar. The executions are triggered as long as the condition $f_{i}\left(e_{i}(k), \delta_{i}\right)>0$ is satisfied. Therefore, the sequence of event triggering instants $0 \leq s_{0}^{i} \leq s_{1}^{i} \leq \cdots \leq s_{l}^{i} \leq \cdots$ is determined iteratively by

$$
s_{l+1}^{i}=\min \left\{k \in \mathbb{N} \mid k>s_{l}^{i}, f_{i}\left(e_{i}(k), \delta_{i}\right)>0\right\} .
$$

Bearing in mind the event-based communication strategy, we can solve the distributed filtering problem via the following steps. Firstly, the sensors synchronically measure the system state according to a time sequence $\{k h\}(k=0,1, \cdots, \infty)$ where $h$ is the sampling interval of the sensor nodes. Secondly, the event generator constructed in each sensor system only utilizes its local messages to check whether the triggering rule is fulfilled. Thirdly, if the triggering condition is satisfied, the sensor node transmits the estimation information, namely, $r_{i}(k)$, to its adjacent nodes via wireless networks. Here, all the sensor nodes keep monitoring the wireless network and, as soon as receiving broadcasts or triggering pre-defined events, the corresponding sensor nodes update the input of local filters.

Remark 1: From the event generator function (4)-(5), it is indicated that once the variation of the difference $e_{i}(k)$ is intense enough and overweighs a certain threshold, the node has to inform all its neighboring sensor nodes of the newest messages so as to keep a satisfactory estimation performance of the wireless sensor network.

Remark 2: Note that the threshold $\delta_{i}$ is a parameter that regulates the triggering frequency. The smaller threshold means the higher frequency of event triggering. When $\delta_{i}=0(i=1,2, \cdots, n)$, the event-based approach reduces to a time-driven one, which requires the sensor nodes to broadcast their information at every sampling instant.

For simplicity, let us consider the time period $k \in\left[s_{l}^{i}, s_{l+1}^{i}\right)$. In the sequel, the event-based filter structures are adopted as follows:

$$
\hat{x}_{i}(k+1)=A_{k} \hat{x}_{i}(k)+\sum_{j \in N_{i}} a_{i j} G_{i j, k} r_{j}^{t}(k),
$$

for $i=1,2, \ldots, n, \quad k \in\left[s_{l}^{i}, s_{l+1}^{i}\right)$, where $G_{i j, k}$ is the filter parameter to be designed. 
It is worth mentioning that the transmission interval in the eventbased schemes is usually larger than that in the periodic transmission schemes. Obviously, compared with existing filtering schemes, the event-based method has its inherently attractive features of reducing the burden of wireless network as well as the energy consumption of sensor systems, thereby helping relieve the network congestion and increase the lifespan of the batteries in sensor nodes.

Remark 3: Due to the distributed nature of the filter algorithm, each sensor node could only access the local estimation and the messages of its neighbors at event triggering instants. Hence, for sensor node $i$, the current filtering messages $r_{j}(k)\left(j \in N_{i}\right)$ remain unavailable but, instead, we could take into account the latest updated values from its adjacent nodes, i.e. $r_{j}^{t}(k)\left(j \in N_{i}\right)$.

The dynamics of the estimation error

$$
\tilde{x}_{i}(k+1)=x_{i}(k+1)-\hat{x}_{i}(k+1),
$$

can be obtained from (1), (2) and (6) as follows

$$
\begin{aligned}
\tilde{x}_{i}(k+1)= & A_{k} \tilde{x}_{i}(k)-\sum_{j \in N_{i}} a_{i j} G_{i j, k} C_{j, k} \tilde{x}_{j}(k)+B_{k} w(k) \\
& -\sum_{j \in N_{i}} a_{i j} G_{i j, k} e_{j}(k)-\sum_{j \in N_{i}} a_{i j} G_{i j, k} D_{j, k} v(k) .
\end{aligned}
$$

For the whole sensor network, we rewrite (7) in a more compact form and arrive at the following augmented filtering error system:

$$
\begin{aligned}
\tilde{x}(k+1)= & \left(\mathcal{A}_{k}-\sum_{i=1}^{n} E_{i} G_{k} H_{i} \mathcal{C}_{k}\right) \tilde{x}(k)-\sum_{i=1}^{n} E_{i} G_{k} H_{i} e(k) \\
& -\sum_{i=1}^{n} E_{i} G_{k} H_{i} \mathcal{D}_{k} \vartheta(k)+\mathcal{B}_{k} \omega(k),
\end{aligned}
$$

where

$$
\begin{aligned}
& \tilde{x}(k)=\operatorname{col}_{n}\left\{\tilde{x}_{i}(k)\right\}, \quad e(k)=\operatorname{col}_{n}\left\{e_{i}(k)\right\}, \omega(k)=\operatorname{col}_{n}\{w(k)\}, \\
& \vartheta(k)=\operatorname{col}_{n}\{v(k)\}, \quad G_{k}=\left\{G_{i j, k}\right\} n \times n, \mathcal{A}_{k}=\operatorname{diag}_{n}\left\{A_{k}\right\}, \\
& \mathcal{B}_{k}=\operatorname{diag}_{n}\left\{B_{k}\right\}, \quad \mathcal{C}_{k}=\operatorname{diag}_{n}\left\{C_{i, k}\right\}, \mathcal{D}_{k}=\operatorname{diag}_{n}\left\{D_{i, k}\right\}, \\
& H_{i}=\operatorname{diag}\left\{a_{i 1} I, \ldots, a_{i n} I\right\}, \quad E_{i}=\operatorname{diag}\{\underbrace{0, \ldots, 0}_{i-1}, I, \underbrace{0, \ldots, 0}_{n-i}\} .
\end{aligned}
$$

The aim of the addressed distributed filtering problem is to design the filter parameters, $G_{i j, k}$, in the filter (6) such that the filtering error covariance is bounded and such a bound is subsequently minimized iteratively by means of Riccati-like difference equations.

\section{MAIn Results}

In this section, we will first obtain an upper bound for the estimation error covariance of the system (8) and then look for an appropriate filter parameter $G_{k}$ to minimize such an upper bound at each time-step $k$.

For presentation convenience, we denote

$$
\begin{aligned}
P_{0}:=\mathbb{E}\left\{\tilde{x}(0) \tilde{x}^{T}(0)\right\}, & P_{k}:=\mathbb{E}\left\{\tilde{x}(k) \tilde{x}^{T}(k)\right\}, \\
\mathcal{Q}_{k}:=\mathbb{E}\left\{\omega(k) \omega^{T}(k)\right\}, & \mathcal{R}_{k}:=\mathbb{E}\left\{\vartheta(k) \vartheta^{T}(k)\right\} .
\end{aligned}
$$

Before proceeding further, we need to introduce the following lemma that will be utilized in the subsequent analysis.

Lemma 1: For $0 \leq k \leq N$, suppose that $X=X^{T} \geq 0, Y=$ $Y^{T} \geq 0$ and $\mathscr{X}_{k}():. \mathbb{R}^{n n_{x} \times n n_{x}} \rightarrow \mathbb{R}^{n n_{x} \times n n_{x}}$. If

$$
\mathscr{X}_{k}(X) \leq \mathscr{X}_{k}(Y), \quad \forall X \leq Y,
$$

then the solutions $W_{k+1}$ and $M_{k+1}$ to the following difference equations

$$
W_{k+1}=\mathscr{X}_{k}\left(W_{k}\right), \quad M_{k+1} \leq \mathscr{X}_{k}\left(M_{k}\right), \quad M_{0}=W_{0}
$$

satisfy

$$
M_{k+1} \leq W_{k+1}
$$

Proof: It follows from (10) that $M_{0} \leq W_{0}$. Assuming, inductively, that $M_{k} \leq W_{k}$, we have

$$
M_{k+1} \leq \mathscr{X}_{k}\left(M_{k}\right) \leq \mathscr{X}_{k}\left(W_{k}\right)=W_{k+1}
$$

The inductive hypothesis implies that $M_{k+1} \leq W_{k+1}$ is always true, which ends the proof.

Before deriving the upper bound for the filtering error variance, let us define the event indicator variables $\beta_{i}(k)$, which takes binary values 0 and 1 , as follows: $\beta_{i}(k)=0$ if the event generator conditions are satisfied at the current instant $k$ for node $i$, while $\beta_{i}(k)=1$ if no event is triggered. Furthermore, denote

$$
\tilde{\beta}_{k}=\operatorname{diag}_{n}\left\{\beta_{i}(k) I_{n_{v}}\right\}, \bar{\beta}_{k}=I_{n n_{v}}-\operatorname{diag}_{n}\left\{\beta_{i}(k) I_{n_{v}}\right\},
$$

which, according to the triggering condtion (4)-(5), should be available at the current instant $k$ for the purpose of calculating the filtering error covariance at the instant $k+1$.

Theorem 1: Consider the linear time-varying system (1) with the distributed filters (6) and event generator condition (4)-(5). Let $\alpha$ be a positive scalar. For $0 \leq k \leq N-1$, assume that there exist two sets of real-valued matrices $\Xi_{k}>0$ and $G_{k}$ satisfying the following Riccatilike difference equation with the initial condition $\Xi_{0}=P_{0} \geq 0$ :

$$
\begin{aligned}
\Xi_{k+1} & =\mathscr{X}_{k}\left(\Xi_{k}\right) \\
& :=(1+\alpha)\left(\mathcal{A}_{k}-\sum_{i=1}^{n} E_{i} G_{k} H_{i} \mathcal{C}_{k}\right) \Xi_{k}\left(\mathcal{A}_{k}-\sum_{i=1}^{n} E_{i} G_{k} H_{i} \mathcal{C}_{k}\right)^{T} \\
& +\Delta\left(1+\alpha^{-1}\right)\left(\sum_{i=1}^{n} E_{i} G_{k} H_{i}\right)\left(\sum_{i=1}^{n} E_{i} G_{k} H_{i}\right)^{T}+\mathcal{B}_{k} \mathcal{Q}_{k} \mathcal{B}_{k}^{T} \\
& +\left(\sum_{i=1}^{n} E_{i} G_{k} H_{i} \mathcal{D}_{k}\right)\left(\bar{\beta}_{k} \mathcal{R}_{k} \bar{\beta}_{k}-\tilde{\beta}_{k} \mathcal{R}_{k} \tilde{\beta}_{k}\right)\left(\sum_{i=1}^{n} E_{i} G_{k} H_{i} \mathcal{D}_{k}\right)^{T}
\end{aligned}
$$

where $\Delta=\sum_{i=1}^{n} \delta_{i}$. Then, the matrix $\Xi_{k}$ is the upper bound of the estimation error covariance matrix $P_{k}$, that is, $\Xi_{k} \geq P_{k}$.

Proof: The estimation error covariance at time-step $k+1$ is computed as follows:

$$
\begin{aligned}
P_{k+1} & =\mathbb{E}\left[\tilde{x}(k+1) \tilde{x}^{T}(k+1)\right] \\
& =\left(\mathcal{A}_{k}-\sum_{i=1}^{n} E_{i} G_{k} H_{i} \mathcal{C}_{k}\right) \mathbb{E}\left\{\tilde{x}(k) \tilde{x}^{T}(k)\right\}\left(\mathcal{A}_{k}-\sum_{i=1}^{n} E_{i} G_{k} H_{i}\right. \\
& \left.\times \mathcal{C}_{k}\right)^{T}+\left(\sum_{i=1}^{n} E_{i} G_{k} H_{i}\right) \mathbb{E}\left\{e(k) e^{T}(k)\right\}\left(\sum_{i=1}^{n} E_{i} G_{k} H_{i}\right)^{T} \\
& +\left(\sum_{i=1}^{n} E_{i} G_{k} H_{i} \mathcal{D}_{k}\right) \mathbb{E}\left\{\vartheta(k) \vartheta^{T}(k)\right\}\left(\sum_{i=1}^{n} E_{i} G_{k} H_{i} \mathcal{D}_{k}\right)^{T} \\
& +\mathcal{B}_{k} \mathbb{E}\left\{\omega(k) \omega^{T}(k)\right\} \mathcal{B}_{k}^{T}+\mathscr{P}_{k}+\mathscr{P}_{k}^{T}+\mathscr{L}_{k}+\mathscr{L}_{k}^{T} \\
& +\mathscr{R}_{k}+\mathscr{R}_{k}^{T}+\mathscr{Q}_{k}+\mathscr{Q}_{k}^{T}+\mathscr{T}_{k}+\mathscr{T}_{k}^{T}+\mathscr{S}_{k}+\mathscr{S}_{k}^{T}
\end{aligned}
$$


where

$$
\begin{aligned}
& \mathscr{P}_{k}=-\left(\mathcal{A}_{k}-\sum_{i=1}^{n} E_{i} G_{k} H_{i} \mathcal{C}_{k}\right) \mathbb{E}\left\{\tilde{x}(k) e^{T}(k)\right\}\left(\sum_{i=1}^{n} E_{i} G_{k} H_{i}\right)^{T}, \\
& \mathscr{L}_{k}=-\left(\mathcal{A}_{k}-\sum_{i=1}^{n} E_{i} G_{k} H_{i} \mathcal{C}_{k}\right) \mathbb{E}\left\{\tilde{x}(k) \vartheta^{T}(k)\right\}\left(\sum_{i=1}^{n} E_{i} G_{k} H_{i} \mathcal{D}_{k}\right)^{T}, \\
& \mathscr{R}_{k}=\left(\mathcal{A}_{k}-\sum_{i=1}^{n} E_{i} G_{k} H_{i} \mathcal{C}_{k}\right) \mathbb{E}\left\{\tilde{x}(k) \omega^{T}(k)\right\} \mathcal{B}_{k}^{T}, \\
& \mathscr{Q}_{k}=\left(\sum_{i=1}^{n} E_{i} G_{k} H_{i}\right) \mathbb{E}\left\{e(k) \vartheta^{T}(k)\right\}\left(\sum_{i=1}^{n} E_{i} G_{k} H_{i} \mathcal{D}_{k}\right)^{T}, \\
& \mathscr{T}_{k}=-\left(\sum_{i=1}^{n} E_{i} G_{k} H_{i}\right) \mathbb{E}\left\{e(k) \omega^{T}(k)\right\} \mathcal{B}_{k}^{T}, \\
& \mathscr{S}_{k}=-\left(\sum_{i=1}^{n} E_{i} G_{k} H_{i} \mathcal{D}_{k}\right) \mathbb{E}\left\{\vartheta(k) \omega^{T}(k)\right\} \mathcal{B}_{k}^{T} .
\end{aligned}
$$

It follows immediately from

$$
\begin{aligned}
& \mathbb{E}\left\{\tilde{x}(k) \vartheta^{T}(k)\right\}=0, \quad \mathbb{E}\left\{\tilde{x}(k) \omega^{T}(k)\right\}=0, \\
& \mathbb{E}\left\{e(k) \omega^{T}(k)\right\}=0, \quad \mathbb{E}\left\{\vartheta(k) \omega^{T}(k)\right\}=0,
\end{aligned}
$$

that $\mathscr{L}_{k}=0, \mathscr{R}_{k}=0, \mathscr{T}_{k}=0, \mathscr{S}_{k}=0$. However, different from the traditional filtering problems, the expectations of some cross terms in $\mathscr{P}_{k}$ and $\mathscr{Q}_{k}$ are no longer zero, which requires further investigations.

First, recall the definition of the gap $e_{i}(k)=r_{i}^{t}(k)-r_{i}(k)$. For the case that the current sampling instant happens to be the event triggering instant of the $i$ th node, namely, $k=s_{l}^{i}$, it is obvious that $e_{i}(k)=0$ and therefore $\mathbb{E}\left\{e_{i}(k) v^{T}(k)\right\}=0$. Otherwise, as $e_{i}(k)=r_{i}^{t}(k)-\left(C_{i, k} \tilde{x}(k)+D_{i, k} v(k)\right)$, we have $\mathbb{E}\left\{e_{i}(k) v^{T}(k)\right\}=$ $\mathbb{E}\left\{\left[r_{i}^{t}(k)-\left(C_{i, k} \tilde{x}(k)+D_{i, k} v(k)\right)\right] v^{T}(k)\right\}=-D_{i, k} R_{k}$. It can now be concluded that

$$
\mathbb{E}\left\{e(k) \vartheta^{T}(k)\right\}=-\mathcal{D}_{k} \tilde{\beta}_{k} \mathcal{R}_{k},
$$

where $\mathcal{R}_{k}=\left[R_{k}\right]_{n \times n}$. Subsequently, we have

$$
\begin{aligned}
\mathscr{Q}_{k}+\mathscr{Q}_{k}^{T} & =\left(\sum_{i=1}^{n} E_{i} G_{k} H_{i} \mathcal{D}_{k}\right)\left[\bar{\beta}_{k} \mathcal{R}_{k} \bar{\beta}_{k}-\tilde{\beta}_{k} \mathcal{R}_{k} \tilde{\beta}_{k}-\mathcal{R}_{k}\right] \\
& \times\left(\sum_{i=1}^{n} E_{i} G_{k} H_{i} \mathcal{D}_{k}\right)^{T} .
\end{aligned}
$$

In the next step, by using the elementary inequality $\left(\alpha^{\frac{1}{2}} M-\right.$ $\left.\alpha^{-\frac{1}{2}} N\right)\left(\alpha^{\frac{1}{2}} M-\alpha^{-\frac{1}{2}} N\right)^{T} \geqslant 0$ where $M$ and $N$ are matrices with compatible dimensions, it follows from (16) that

$$
\begin{aligned}
\mathscr{P}_{k} & +\mathscr{P}_{k}^{T} \leq \alpha\left(\mathcal{A}_{k}-\sum_{i=1}^{n} E_{i} G_{k} H_{i} \mathcal{C}_{k}\right) \mathbb{E}\left\{\tilde{x}(k) \tilde{x}^{T}(k)\right\}\left(\mathcal{A}_{k}-\sum_{i=1}^{n} E_{i}\right. \\
& \left.\times G_{k} H_{i} \mathcal{C}_{k}\right)+\alpha^{-1}\left(\sum_{i=1}^{n} E_{i} G_{k} H_{i}\right) \mathbb{E}\left\{e(k) e^{T}(k)\right\}\left(\sum_{i=1}^{n} E_{i} G_{k} H_{i}\right)^{T}
\end{aligned}
$$

In addition, under the event-based strategy, the gap $e_{i}(k)$ would be immediately reset to zero if the triggering condition are fulfilled. Consequently, the following inequality is always satisfied:

$$
e^{T}(k) e(k) \leq \Delta,
$$

where $\Delta=\sum_{i=1}^{n} \delta_{i}$. Applying the properties of matrix operations, we obtain

$$
e(k) e^{T}(k) \leq\|e(k)\|^{2} I=e^{T}(k) e(k) I \leq \Delta I,
$$

and hence

$$
\mathbb{E}\left\{e(k) e^{T}(k)\right\} \leq \Delta I
$$

Now, it follows from (17), (19), (20) and (23) that

$$
P_{k+1} \leq \mathscr{X}_{k}\left(P_{k}\right)
$$

Define a positive semidefinite matrix $\Xi_{0}=P_{0}$, and $\Xi_{k+1}$ can be calculated iteratively by the Riccati-like difference equation:

$$
\Xi_{k+1}=\mathscr{X}_{k}\left(\Xi_{k}\right) .
$$

Obviously, the conditions in Lemma 1 are satisfied. Therefore, for all $0 \leq k \leq N$, we have

$$
P_{k+1} \leq \Xi_{k+1}
$$

and the proof is now complete.

Remark 4: It is noticed that, in (14), the term $\left(\sum_{i=1}^{n} E_{i} G_{k} H_{i}\right)\left(\sum_{i=1}^{n} E_{i} G_{k} H_{i}\right)^{T} \geq 0$. Therefore, the upper bound will increase as the threshold $\Delta$ increases. From the engineering viewpoint, a larger threshold would lead to a fewer number of transmissions over the network, which implies that the threshold $\Delta$ does have a major impact on the tradeoffs between the filtering performance and the sensor data transmission rate.

Remark 5: In the recursive calculation of the performance index, namely, the filtering error covariance, the triggering information at the time instant $k$ is explicitly exploited in (14) in terms of the binary variable $\beta_{i}(k)$. This would definitely help reduce the conservatism and tighten the upper bound. Comparing to the traditional eventtriggering mechanism where only the stability is the concern, the introduction of such a binary variable $\beta_{i}(k)$ would play an important role in estimating the filtering performance (i.e., the error covariance).

Remark 6: In the case of the threshold $\delta_{i}=0$, it is not difficult to see that the triggering rules are always fulfilled for every sensor nodes and the indicator $\tilde{\beta}_{k}$ is a zero matrix with appropriate dimensions. Consequently, the Riccati-like difference equation (14) would reduce to the recursion of the estimation error covariance for the traditional distributed filtering problem.

We are now ready to minimize the upper bound $\Xi_{k}$ on each recursion by appropriately designing the filter parameters. For this purpose, the following lemma is useful.

Lemma 2: For matrices $M, X, N, L$ with compatible dimensions, the following are true:

$$
\begin{aligned}
& \frac{\partial}{\partial X} \operatorname{tr}\left(M X^{T}\right)=M, \quad \frac{\partial}{\partial X} \operatorname{tr}(X M)=M^{T}, \\
& \frac{\partial}{\partial X} \operatorname{tr}(M X N)=M^{T} N^{T}, \quad \frac{\partial}{\partial X} \operatorname{tr}\left(M X^{T} N\right)=N M, \\
& \frac{\partial}{\partial X} \operatorname{tr}\left(M X N X^{T} L\right)=M^{T} L^{T} X N^{T}+L M X N .
\end{aligned}
$$

Furthermore, for any symmetric matrix $P$, the following holds

$$
\left.\frac{\partial}{\partial X} \operatorname{tr}\left\{(M X N) P(M X N)^{T}\right)\right\}=2 M^{T} M X N P N^{T} .
$$

For presentation simplicity, we denote

$$
\begin{aligned}
\mathcal{A}_{k}^{(i)}= & \{\underbrace{0, \ldots, 0}_{i-1}, A_{k}, \underbrace{0, \ldots, 0}_{n-i}\}, \\
G_{k}= & \left\{G_{i j, k}\right\}_{n \times n}, \quad G_{k}^{(i)}=\left\{G_{i 1, k}, G_{i 2, k}, \cdots, G_{i n, k}\right\}, \\
\mathcal{M}_{i, k}= & (1+\alpha) H_{i} \mathcal{C}_{k} \Xi_{k}\left(H_{i} \mathcal{C}_{k}\right)^{T}+\Delta\left(1+\alpha^{-1}\right) H_{i} H_{i}, \\
& +H_{i} \mathcal{D}_{k}\left(\bar{\beta}_{k} \mathcal{R}_{k} \bar{\beta}_{k}-\tilde{\beta}_{k} \mathcal{R}_{k} \tilde{\beta}_{k}\right)\left(H_{i} \mathcal{D}_{k}\right)^{T}, \\
\mathcal{N}_{i, k}= & (1+\alpha) \mathcal{A}_{k}^{(i)} \Xi_{k}\left(H_{i} \mathcal{C}_{k}\right)^{T} .
\end{aligned}
$$

Furthermore, let $\bar{G}_{k}^{(i)}$ and $\overline{\mathcal{N}}_{i, k}$ be the simplified matrices by removing the $j$ th $\left(j \notin N_{i}\right)$ column from $G_{k}^{(i)}$ and $\mathcal{N}_{i, k}$, respectively. Also, let $\overline{\mathcal{M}}_{i, k}$ be a simplified matrix by removing both the $j$ th $\left(j \notin N_{i}\right)$ row and $j$ th $\left(j \notin N_{i}\right)$ column from $\mathcal{M}_{i, k}$. 
Theorem 2: Consider the linear time-varying system (1) with the distributed filters (6) and event generator condition (4)-(5). The solution $\Xi_{k}$ to (14), which is an upper bound of the estimation error covariance matrix $P_{k}$, can be minimized at each iteration with the filter parameter $G_{k}=\left\{G_{i j, k}\right\}_{n \times n}$ given by

$$
\bar{G}_{k}^{(i)}=\overline{\mathcal{N}}_{i, k} \overline{\mathcal{M}}_{i, k}^{-1}, \text { for all } 0 \leq i \leq n, 0 \leq k \leq N-1,
$$

and $G_{i j, k}=0$ for $j \notin N_{i}$. Here, $\bar{G}_{k}^{(i)}, \overline{\mathcal{N}}_{i, k}$ and $\overline{\mathcal{M}}_{i, k}$ are defined right after (29).

Proof: According to (14), one has

$$
\begin{aligned}
& \operatorname{tr}\left(\Xi_{k+1}\right)=(1+\alpha) \operatorname{tr}\left\{\mathcal{A}_{k} \Xi_{k} \mathcal{A}_{k}^{T}+\left(\sum_{i=1}^{n} E_{i} G_{k} H_{i} \mathcal{C}_{k}\right) \Xi_{k}\left(\sum_{i=1}^{n} E_{i} G_{k}\right.\right. \\
& \left.\left.\times H_{i} \mathcal{C}_{k}\right)^{T}-\mathcal{A}_{k} \Xi_{k}\left(\sum_{i=1}^{n} E_{i} G_{k} H_{i} \mathcal{C}_{k}\right)^{T}-\left(\sum_{i=1}^{n} E_{i} G_{k} H_{i} \mathcal{C}_{k}\right) \Xi_{k} \mathcal{A}_{k}^{T}\right\} \\
& +\Delta\left(1+\alpha^{-1}\right) \operatorname{tr}\left\{\left(\sum_{i=1}^{n} E_{i} G_{k} H_{i}\right)\left(\sum_{i=1}^{n} E_{i} G_{k} H_{i}\right)^{T}\right\}+\operatorname{tr}\left\{\mathcal{B}_{k} \mathcal{Q}_{k} \mathcal{B}_{k}^{T}\right. \\
& \left.\left(\sum_{i=1}^{n} E_{i} G_{k} H_{i} \mathcal{D}_{k}\right)\left(\bar{\beta}_{k} \mathcal{R}_{k} \bar{\beta}_{k}-\tilde{\beta}_{k} \mathcal{R}_{k} \tilde{\beta}_{k}\right)\left(\sum_{i=1}^{n} E_{i} G_{k} H_{i} \mathcal{D}_{k}\right)^{T}\right\}
\end{aligned}
$$

Resorting to the properties of trace, we have

$$
\operatorname{tr}\left\{E_{i} M \Xi_{k}\left(E_{j} N\right)^{T}\right\}=0 \text {, if } i \neq j,
$$

where $M$ and $N$ are matrices with appropriate dimensions. Next, taking the partial derivation of the trace of the matrix $\Xi_{k+1}$ with respect to the parameters $G_{k}$, and utilizing the equation (27) and (28), we arrive at

$$
\begin{aligned}
& \frac{\partial}{\partial G_{k}} \operatorname{tr}\left\{\Xi_{k+1}\right\}=2(1+\alpha)\left\{\sum_{i=1}^{n} E_{i} G_{k} H_{i} \mathcal{C}_{k} \Xi_{k}\left(H_{i} \mathcal{C}_{k}\right)^{T}-\sum_{i=1}^{n} E_{i}\right. \\
& \left.\quad \times \mathcal{A}_{k} \Xi_{k}\left(H_{i} \mathcal{C}_{k}\right)^{T}\right\}+2 \Delta\left(1+\alpha^{-1}\right) \sum_{i=1}^{n} E_{i} G_{k} H_{i} H_{i} \\
& \quad+2 \sum_{i=1}^{n} E_{i} G_{k} H_{i} \mathcal{D}_{k}\left(\bar{\beta}_{k} \mathcal{R}_{k} \bar{\beta}_{k}-\tilde{\beta}_{k} \mathcal{R}_{k} \tilde{\beta}_{k}\right)\left(H_{i} \mathcal{D}_{k}\right)^{T}=0 .
\end{aligned}
$$

Subsequently, as $G_{k}^{(i)}$ and $\mathcal{A}_{k}^{(i)}$ represent, respectively, the $i$ th row of matrices $G_{k}$ and the $i$ th row of $\mathcal{A}_{k}$, we have

$$
G_{k}^{(i)} \mathcal{M}_{i, k}=\mathcal{N}_{i, k}
$$

Recalling the definition $H_{i}=\operatorname{diag}\left\{a_{i 1} I, a_{i 2} I,, \ldots, a_{i n} I\right\}$ and the relation $a_{i j}=0\left(j \notin N_{i}\right)$, one can remove the corresponding zero rows and zero columns from $\mathcal{M}_{i, k}$ and the corresponding zero columns from $\mathcal{N}_{i, k}$ to obtain the simplified matrices $\overline{\mathcal{M}}_{i, k}$ and $\overline{\mathcal{N}}_{i, k}$. Let $\mathcal{M}_{i, k}=\left\{\tilde{M}_{a b, k}\right\}_{n \times n}$ and $\mathcal{N}_{i, k}=\left\{\tilde{N}_{b, k}\right\}_{1 \times n}$. If $b \in N_{i}$, it follows that

$$
\sum_{j=1}^{n} G_{i j, k} \tilde{M}_{j b, k}=\tilde{N}_{b, k} .
$$

In other cases, the above equation is always satisfied since both sides of it are zero. Because $G_{i j, k}, j \notin N_{i}$ are chosen as zero matrices of appropriate dimensions and the corresponding rows of $\mathcal{M}_{i, k}$ are also zeros, it can be seen that

$$
\bar{G}_{k}^{(i)} \overline{\mathcal{M}}_{i, k}^{\left(b^{\prime}\right)}=\overline{\mathcal{N}}_{i, k}^{\left(b^{\prime}\right)},
$$

where $\bar{G}_{k}^{(i)}$ is a simplified matrix of $G_{k}^{(i)}$ by removing $G_{i j, k}, j \notin N_{i}$, $b^{\prime}$ is the new column index of the simplified matrix, and $\overline{\mathcal{M}}_{i, k}^{\left(b^{\prime}\right)}, \mathcal{N}_{i, k}^{\left(b^{\prime}\right)}$ are the $b^{\prime}$ th column of matrices $\overline{\mathcal{M}}_{i, k}, \overline{\mathcal{N}}_{i, k}$, respectively. It is not difficult to verify that the matrix $\overline{\mathcal{M}}_{i, k}$ is positive definite and the filter parameters can be calculated as follows:

$$
\bar{G}_{k}^{(i)}=\overline{\mathcal{N}}_{i, k} \overline{\mathcal{M}}_{i, k}^{-1}
$$

which completes the proof.

Remark 7: In Theorem 2, a novel matrix simplification technique is developed to handle the challenges resulting from the sparseness of the sensor network topology and preserving structure of the distributed filters. By using the employed SoD principle, the executions are triggered when certain signal exceeds a time-independent threshold defined as an important change of its value, and therefore the communication load and energy consumption in practice can be alleviated by avoiding unnecessary transmissions.

Remark 8: In the time-invariant case, all system parameters are constant matrices and the boundedness problem of $\Xi_{k}$ can be dealt with by examining the Schur stability of the matrix $(1+\alpha)^{1 / 2}(\mathcal{A}-$ $\left.\sum_{i=1}^{n} E_{i} G H_{i} \mathcal{C}\right)$. In this case, by properly designing the filter parameter, the estimate error can be guaranteed to be bounded.

Remark 9: In this paper, the distributed filtering problem is solved for a class of discrete time-varying systems with an event-based communication mechanism. By using an event indicator variable, the triggering information is included in the Riccati-like difference equation whose solution serves as an upper bound for the filtering error covariance which is, subsequently, minimized by appropriately designing the filter parameters iteratively. Note that the main result established in Theorem 2 contains all the information about the system parameters, the topology information as well as the triggering information.

\section{An ILlustrative EXAMPLE}

To illustrate the validity of the proposed filter design strategy, we consider a second-order system (1) where $x(k)=\left(x_{1}(k) x_{2}(k)\right)^{T} \in$ $\mathbb{R}^{2}$, the random noise $w(k)$ is a zero-mean Gaussian sequences with covariance $Q_{k}=1$, and

$$
\begin{aligned}
& A_{k}=\left[\begin{array}{cc}
0.98+0.05 \sin (0.12 k) & 0.4 \\
0.15 & -0.75
\end{array}\right], \\
& B_{k}=\left[\begin{array}{ll}
0.16 & 0.18
\end{array}\right]^{T} .
\end{aligned}
$$

Suppose the initial value of the state $x(0)$ is uniformly distributed over the region $[-6,6]$ so that $\mathbb{E} x(0)=\left[\begin{array}{ll}0 & 0\end{array}\right]$.

The sensor network is represented by a directed graph $\mathcal{G}=$ $(\mathcal{V}, \mathcal{E}, \mathcal{A})$ where the set of nodes $\mathcal{V}=\{1,2,3,4\}$, the set of edges $\mathcal{E}=\{(1,1),(1,2),(2,1),(2,2),(2,3),(3,1),(3,3),(4,1),(4,4)\}$, and the adjacency elements associated with the edges of the graph are $a_{i j}=1$. The dynamics of the sensor nodes is modeled as (2) with

$$
\begin{aligned}
& C_{1, k}=[0.82,0.62+0.05 \cos (0.12 k)], \\
& C_{2, k}=[0.75+0.05 \sin (0.1 k), 0.80] \text {, } \\
& C_{3, k}=[0.74+0.05 \sin (0.1 k), 0.75+0.05 \cos (0.1 k)] \text {, } \\
& C_{4, k}=[0.75,0.65] \text {. }
\end{aligned}
$$

In the simulation, let the measurement noise $v(k)$ be a zero-mean Gaussian white noise sequences with covariance $R_{k}=1$. Choose the thresholds $\delta_{i}=0.4$ for $i=1,2,3,4$ and the scalar $\alpha=0.1$. The initial estimations about the plant state are $\hat{x}_{1}(0)=\hat{x}_{2}(0)=\hat{x}_{3}(0)=$ $\hat{x}_{4}(0)=\left[\begin{array}{ll}0 & 0\end{array}\right]$. The trace of mean square error for the estimation of the state is averaged in 500 runs of simulation. The suboptimal upper bound and corresponding filter parameters $G_{k}$ can be calculated at each iteration according to (14) and (30).

Simulation results are shown in Figs. 1-4. In Fig. 1 and Fig. 2, the trajectories for the states and respective estimates are depicted, from which we can see that the proposed strategy has a favorable performance in estimating the state of a divergent system. Besides, compared with time-driven mechanism, the broadcast times are significantly reduced in Fig. 3, which embodies the superiority of 

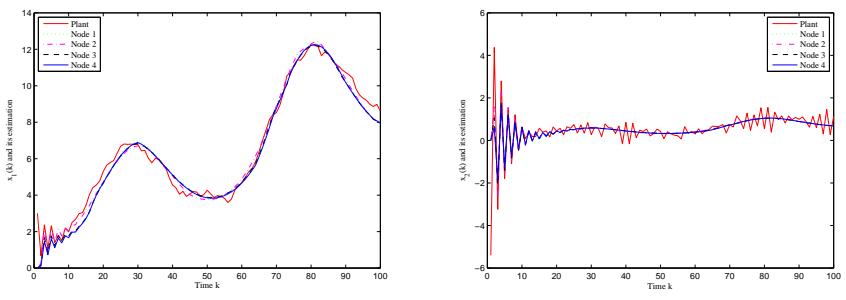

Fig. 1. The state $x_{1}$ and its estimation Fig. 2. The state $x_{2}$ and its estimation

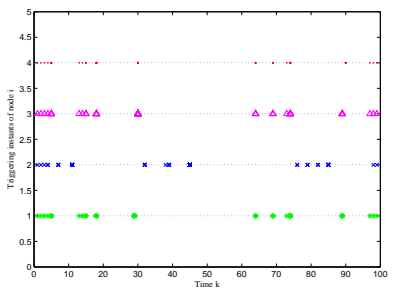

Fig. 3. The triggering sequence

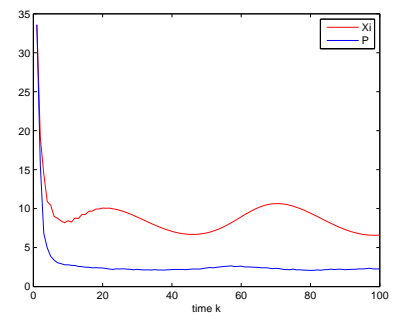

Fig. 4. The trace of error covariance and its upper boundary the event-based one. To this end, Fig. 4 shows the upper bound $\Xi_{k}$ of the error covariance matrix $P_{k}$.

To evaluate the influence of event thresholds and noise covariances on the communication rate (total number of communications in the network divided by running time), we have the following experimental results via 500 trials:

TABLE I

THE INFLUENCE OF THRESHOLDS

\begin{tabular}{ccccc}
\hline$\sigma_{i}, i=1, \ldots, 4$ & 0.1 & 0.2 & 0.4 & 0.8 \\
\hline Average Communication Rate & 1.8908 & 1.3328 & 0.8160 & 0.5316 \\
\hline
\end{tabular}

TABLE II

THE INFLUENCE OF NOISE COVARIANCES

\begin{tabular}{ccccc}
\hline$\left(Q_{k}, R_{k}\right)$ & $(0.5,0.5)$ & $(1,1)$ & $(2,2)$ & $(4,4)$ \\
\hline Average Communication Rate & 0.3920 & 0.8160 & 1.9164 & 2.8608 \\
\hline
\end{tabular}

Obviously, both the decrease of thresholds and the increase of noise covariances would contribute to a higher communication frequency.

\section{Conclusions}

In this paper, a novel event-based distributed filter has been proposed in wireless sensor networks for the sake of reducing the sensor data transmission rate and the energy consumption. Basing on a SoD concept, each smart sensor node makes decisions independently about when the local messages have to be broadcast. By using a combination of trace properties, inductive method and event-induced behaviors, we have computed the upper bound for the filtering error covariance recursively by solving a Riccati-like matrix equation. Subsequently, the distributed filter gain has been properly designed at every iteration to minimize such an upper bound. Finally, via a numerical example, we have demonstrated the effectiveness of the event-based communication protocol in reducing the number of transmissions over the network.

\section{REFERENCES}

[1] M. Basin, P. Shi, D. Calderon-Alvarez, and J. Wang, Central suboptimal $\mathcal{H}_{\infty}$ filter design for linear time-varying systems with state or measurement delay, Circuits, Systems and Signal Processing, vol. 28, no. 2, pp. 305-330, 2009.
[2] R. Caballero-Aguila, A. Hermoso-Carazo, J. D. Jimenez-Lopez, J. Linares-Perez and S. Nakamori, Signal estimation with multiple delayed sensors using covariance information, Digital Signal Processing, Vol. 20, No. 2, pp. 528-540, 2010.

[3] D. Ding, Z. Wang, B. Shen and H. Shu, $H_{\infty}$ state estimation for discretetime complex networks with randomly occurring sensor saturations and randomly varying sensor delays, IEEE Trans. on Neural Networks, Vol. 23, No. 5, pp. 725-736, May 2012.

[4] D. V. Dimarogonas, E. Frazzoli and K. H. Johansson, Distributed event-triggered control for multi-agent systems, IEEE Trans. Automatic Control, Vol. 57, No. 5, pp. 1291-1297, May 2012.

[5] H. Dong, Z. Wang and H. Gao, Distributed $H_{\infty}$ filtering for a class of Markovian jump nonlinear time-delay systems over lossy sensor networks, IEEE Trans. Industrial Electronics, Vol. 60, No. 10, pp. 46654672, Oct. 2013.

[6] H. Gao, T. Chen, and J. Lam, A new delay system approach to networkbased control, Automatica, vol. 44, no. 1, pp. 39-52, Jan. 2008.

[7] E. Garcia and P. Antsaklis, Model-based event-triggered control for systems with quantization and time-varying network delays, IEEE Trans. Automatic Control, Vol. 58, No. 2, pp. 422-434, Feb. 2013.

[8] S. Havedanloo and H. R. Karimi, Improving the performance metric of wireless sensor networks with clustering Markov chain model and multilevel fusion, Mathematical Problems in Engineering, Art. No. 783543, 2013.

[9] J. Hu, G. Chen and H. X. Li, Distributed event-triggered tracking control of leader-follower multi-agent systems with communication delays, Kybernetika, Vol. 47, No. 4, pp. 630-643, 2011.

[10] S. Hu and D. Yue, Event-based $H_{\infty}$ filtering for networked system with communication delay, Signal Processing, Vol. 92, No. 9, pp. 2029-2039, Sep. 2012.

[11] C. Huang, D. W. C. Ho and J. Lu, Partial-information-based distributed filtering in two-targets tracking sensor networks, IEEE Trans. Circuits and Systems I - Regular Papers, Vol. 59, No. 4, pp. 820-832, Apr. 2012.

[12] J. Liang, B. Shen, H. Dong and J. Lam, Robust distributed state estimation for sensor networks with multiple stochastic communication delays, it Int. J. Systems Science, Vol. 42, No. 9, pp. 1459-1471, 2011.

[13] A. Le and R. McCann, Event-based measurement updating Kalman filter in network control systems, Proc. IEEE Region 5 Technical Conf., Fayetteville, AR, USA, pp. 138-141, Apr. 2007.

[14] Q. Liu, Z. Wang, X. He and D. H. Zhou, A survey of event-based strategies on control and estimation, Systems Science and Control Engineering: An Open Access Journal, Vol. 2, No. 1, pp. 90-97, 2014.

[15] M. Miskowicz, Send-on-delta concept: an event-based data reporting strategy, Sensors, Vol. 6, No. 1, pp. 49-63, Jan. 2006.

[16] V. H. Nguyen and Y. S. Suh, Improving estimation performance in networked control systems applying the send-on-delta transmission method, Sensors, Vol. 7, No. 10, pp. 2128-2138, Oct. 2007.

[17] R. Olfati-Saber, Distributed Kalman filtering for sensor networks, Proc. 46th IEEE Conf. Decision and Control, New Orleans, LA, USA, pp. 5492-5498, Dec. 2007.

[18] J. M. Rabaey, M. J. Ammer, J. L. da Silva, D. Patel and S. Roundy, Picoradio supports ad hoc ultra-low power wireless networking, IEEE Computer, Vol. 33, No. 7, pp. 42-48, Jul. 2000.

[19] B. Shen, Z. Wang and Y. S. Hung, Distributed $H_{\infty}$-consensus filtering in sensor networks with multiple missing measurements: the finite-horizon case, Automatica, Vol. 46, No. 10, pp. 1682-1688, Oct. 2010.

[20] Y. S. Suh, V. H. Nguyen and Y. S. Ro, Modified Kalman filter for networked monitoring systems employing a send-on-delta method, Automatica, Vol. 43, No. 2, pp. 332-338, Feb. 2007.

[21] S. Sun, L. Xie, and W. Xiao, Optimal full-order and reduced-order estimators for discrete-time systems with multiple packet dropouts, IEEE Transactions on Signal Processing, vol. 56, no. 8, pp. 4031-4038, Aug. 2008.

[22] G. Welch and G. Bishop, An introduction to the Kalman filter, Notes of ACM SIGGRAPH tutorial on the Kalman Filter, 2001.

[23] X. Wang and M. Lemmon, Decentralized event-triggered broadcasts over networked control systems, Hybrid Systems: computation and control, Berlin: Springer, pp. 674-677, 2008.

[24] D. Yue, E. Tian and Q. Han, A delay system method for designing event-triggered controllers of networked control systems, IEEE Trans. Automatic Control, Vol. 58, No. 2, pp. 475-481, Feb. 2013.

[25] X. Yin and D. Yue, Event-triggered tracking control for heterogeneous multi-agent systems with Markov communication delays, Journal of the Franklin Institute, Vol. 350, No. 5, pp. 1312-1334, Jun. 2013.

[26] R. Zheng, J. Hou and N. Li, Power management and power control in wireless networks, Ad Hoc and Sensor Networks, New York: Nova Science Publishers, pp. 1-25, 2004 